\title{
PREDICCIÓN DEL BURNOUT EN LOS DOCENTES: PAPEL DE LOS FACTORES ORGANIZACIONALES, PERSONALES Y SOCIODEMOGRÁFICOS
}

\author{
Jesús Esteras, Paloma Chorot y Bonifacio Sandín \\ Facultad de Psicología, Universidad Nacional de Educación a Distancia, Madrid, España
}

\begin{abstract}
Resumen: El propósito del presente estudio consistió en examinar la contribución de las variables organizacionales, personales y demográficas como predictores de los niveles de burnout percibido en una muestra de 171 docentes de instituciones públicas y privadas (preescolar, primaria, y ESO/ bachillerato). Los participantes cumplimentaron cuestionarios sobre satisfacción laboral, estrés laboral y características contextuales del trabajo docente (apoyos de supervisión, falta de reconocimiento profesional, condiciones organizacionales, y preocupaciones profesionales), afectividad, y variables sociodemográficas (edad, sexo, estado civil, religión, años de experiencia, tipo de centro, y etapa educativa). Los resultados basados en análisis de regresión múltiple indicaron que las variables organizacionales se relacionaban de forma diferencial con las dimensiones de burnout, mientras que la afectividad negativa actuaba como factor general de vulnerabilidad. El estrés laboral y la baja satisfacción laboral fueron poderosos predictores del agotamiento emocional. Los resultados delimitan posibles factores protectores y de vulnerabilidad hacia el burnout en los docentes.
\end{abstract}

Palabras clave: Burnout en docentes; estrés laboral; satisfacción laboral; entorno laboral docente; afectividad.

\section{Predicting teacher burnout: The role of organizational, personal and socio-demographic variables}

\begin{abstract}
The purpose of the present study was to examine the contribution of organizational, personal and demographic variables in predicting perceived levels of burnout in a sample of 171 teachers working in public and private education institutions (preschool, elementary school, and middle school). Participants completed measures of job satisfaction, work stress, perceived teacher working context (supervisory support, lack of professional recognition, organizational conditions, and professional worry), affectivity, and socio-demographics (age, sex, marital status, religion, job experience, kind of education institution, and level in teaching). Results of a series of multiple regression analyses indicated that organizational variables were differentially related to dimensions of burnout, whereas negative affect appeared to act as a general predictor of vulnerability. Job stress and low work satisfaction were strong predictors of emotional exhaustion. Results provide relevant information to determine the protective and vulnerability factors as to teacher burnout.
\end{abstract}

Keywords: Teacher burnout; job stress; work satisfaction; teacher working environment; affectivity.

\section{INTRODUCCIÓN}

El concepto de burnout, o síndrome de estar quemado, fue descrito originalmente por Freu-

\footnotetext{
Recibido: 10 julio 2013; aceptado: 1 septiembre 2013.

Correspondencia: Bonifacio Sandín, Universidad Nacional de Educación a Distancia, Facultad de Psicología, Juan del Rosal 10, 28040 Madrid, España. Correo-e: bsandin@, psi.uned.es
}

denberger (1974) para referirse a las reacciones físicas y mentales que observó en un grupo de personas que trabajaban en una clínica de desintoxicación; después de un año, muchos de ellos se sentían agotados, irritables, y habían desarrollado una actitud cínica hacia sus pacientes y una tendencia a evitarlos (Sandín, 2008). Posteriormente Maslach y Jackson (1981) lo definieron como un trastorno adaptativo crónico, asociado a las demandas psicosociales que re- 
quiere el trabajo directo con personas, experimentando un desequilibrio prolongado entre las demandas y los recursos de afrontamiento. El trastorno suele entenderse como una respuesta al estrés laboral que surge tras un proceso de reevaluación cognitiva, cuando las estrategias de afrontamiento empleadas no son eficaces para reducir ese estrés laboral percibido. La mayoría de los trabajos de investigación realizados hasta la fecha están basados en la propuesta conceptual de Maslach y Jackson (1981), los cuales describieron el síndrome de burnout como un estado psicológico de agotamiento, agotamiento emocional y despersonalización asociado a desmotivación, baja autoestima, sentimiento de fracaso y disminución del rendimiento. Tras la publicación por estas autoras del ya clásico cuestionario de autoinforme para la evaluación del burnout (Maslach y Jackson, 1986), se ha venido entendiendo el burnout como un constructo multidimensional constituido por al menos tres dimensiones separadas: agotamiento emocional, despersonalización, y baja realización personal. Algunos autores (p.ej., Pines y Aronson, 1988), sin embargo, mantienen la hipótesis de que el burnout es un constructo unidimensional, caracterizado por el agotamiento, si bien pueden diferenciarse como componentes del burnout la desmoralización y la baja motivación, identificándolo en cierto modo con el concepto de agotamiento vital (véase Sandín, 2008).

La evidencia relacionada con la investigación sobre el burnout tiende a indicar que el burnout es un proceso crónico patológico, que sigue un curso temporal donde van sucediéndose diversas etapas, y cada etapa presenta ciertos síntomas característicos y diferenciadores (Campagne, 2012; Gascón, Olmedo y Ciccote1li, 2003; Gil-Monte y Peiró, 1997; Gómez-Alcaina, Montero-Marín, Demarzo, Pereira y García-Campayo, 2013; Ramos, 1999). Se ha sugerido que la mayor incidencia del burnout se da en los profesionales que ofrecen un servicio asistencial y cuyos destinatarios de su labor profesional son las personas, como ocurre en los profesionales de la educación tales como los profesores, los psicopedagogos y los orientadores (Boydak, 2009).

La profesión docente no se reduce a las funciones didácticas y responsabilidades relacio- nadas con el proceso educativo en sí mismo, sino que implica una dedicación más personal y emocional que le infiere un carácter de profesión vocacional. Este aspecto, unido a la polémica relación que actualmente caracteriza las interacciones entre docentes y discentes, denota que los docentes podrían constituir un grupo particularmente vulnerable a sufrir burnout. Éste puede ser una consecuencia de un estado de estrés mantenido en el tiempo, que se cronifica y se manifiesta por un agotamiento emocional en el docente, el cual puede ir acompañado por una desvinculación o alejamiento afectivo de sus estudiantes y compañeros (despersonalización o cinismo) y por una sensación de baja realización en el trabajo (Schaufeli, Leiter y Maslach, 2009).

Algunos trabajos centrados en el personal docente han estudiado el papel de posibles variables implicadas en el fenómeno del burnout. Se han señalado, en este sentido, variables relacionadas con el entorno laboral, los problemas de disciplina, la desmotivación del alumnado, la escasa autonomía, los cambios sociales y curriculares, la sobrecarga laboral, la masificación de las aulas, la baja participación en las decisiones, los problemas con los directores o inspectores, el pobre reconocimiento económico, las políticas y reiteradas reformas educativas, la formación, la promoción, y el desarrollo profesional deficitario (Burke y Greenglass, 1994; Moriarty, Martín y Blatchford, 2001).

Diversas variables socio-demográficas como el sexo, la edad, el estado civil, la paternidad, las relaciones personales y la etapa educativa donde se imparte docencia, han sido relacionadas tanto con el estrés laboral como con el burnout (Lau, Yuen y Chan, 2005; León-Rubio, León-Pérez y Cantero, 2013; Moreno, Aranda, Aldrete, Flores y Pozo, 2006; Padilla et al., 2009). Se ha señalado, por ejemplo, que las mujeres presentan niveles mayores de cansancio emocional y menores niveles en realización personal que los hombres (Maslach, 1982; Maslach y Jackson, 1981). En contraste, otros autores no han encontrado diferencias significativas entre hombres y mujeres en las dimensiones del burnout (Schwab e Iwanicki, 1982). Purvanova y Muros (2010) realizaron una revisión de 183 trabajos y concluyeron que las mujeres presen- 
tan una tendencia a puntuar alto en los ítems relacionados con el agotamiento emocional, mientras que los hombres lo hacían en los ítems correspondientes a la dimensión de despersonalización. Esta tendencia sugiere una expresión diferenciada según el género en las dimensiones del síndrome de burnout. En esta misma línea se han encontrado otros estudios que parecen confirmar también dicha tendencia en el colectivo de docentes (González-Morales, Rodríguez y Peiró, 2010). Otros estudios, aunque han cuestionado la propensión femenina a puntuar más alto en agotamiento emocional, sí parecen confirmar que los hombres perciben de forma más amenazante su entorno laboral y, en consecuencia, manifiestan mayores niveles en las dimensiones despersonalización y falta de realización personal, presentado mayor prevalencia en burnout que las mujeres (León-Rubio et al., 2013).

Algunos autores han encontrado que las variables familiares como la estabilidad afectiva en las relaciones personales y la paternidad actúan como elementos protectores contra el burnout. Así, a partir del trabajo de Oliver (1993) se concluye que los educadores que no tienen pareja ni tienen hijos son más vulnerables a desarrollar el síndrome de burnout.

Las variables del contexto organizacional han sido referidas como determinantes importantes en el desarrollo del síndrome de burnout, así como también la baja consideración que se tiene sobre la labor docente (Manassero et al., 2005). Los aspectos relacionados directamente con el ámbito organizacional de los centros, como son la falta de consenso, las actitudes y conductas negativas de la dirección, la falta de capacitaciones, etc., son factores que limitan el desarrollo profesional y el desempeño de los docentes, haciéndoles más vulnerables hacia el síndrome de burnout (Briones, Babernos, y Arenas, 2010). Tanto la falta de satisfacción laboral (Durán, Extremera, Montalbán, y Rey, 2005) como la carga laboral (Gil-Monte y Peiró, 1997) parecen estar relacionadas con el agotamiento emocional. Asimismo, se ha sugerido cierta relación entre el nivel docente impartido y el síndrome de burnout, asociándose éste de forma más específica con las etapas educativas superiores (Beer y Beer, 1992).
Las creencias religiosas parecen influir positivamente en la prevención del síndrome de burnout. Algunos estudios han evidenciado que la espiritualidad se convierte en una forma de afrontamiento para las personas (Mytko y Knight, 1999), tal y como se aprecia mediante la inclusión de una dimensión de religión en los recientes instrumentos de evaluación del afrontamiento del estrés (Sandín y Chorot, 2003).

El objetivo del presente estudio ha consistido en examinar en una muestra de personal docente (profesorado de Educación Infantil, Educación Primaria, Educación Secundaria Obligatoria y Bachillerato) la capacidad predictiva de la satisfacción laboral, el estrés laboral y las variables organizacionales (problemática laboral, supervisión, condiciones organizacionales, preocupaciones profesionales, y falta de reconocimiento profesional) sobre las puntuaciones en las dimensiones de burnout, así como también sobre el síndrome de burnout. Así mismo, pretendemos estudiar el posible papel que en este sentido juegan los factores sociodemográficos. Dada la relación que existe entre la afectividad y estas variables (Sandín, 2008), examinamos dichas relaciones controlando el posible efecto del afecto negativo y positivo. Hipotetizamos que las puntuaciones en burnout deberían asociarse negativamente a las puntuaciones en factores protectores como la satisfacción laboral y afecto positivo, y positivamente con las puntuaciones en las variables desadaptativas o de vulnerabilidad personal (afecto negativo) y organizacional (problemática laboral, estrés de rol, supervisión inadecuada, falta de reconocimiento profesional, etc.). Dado que actualmente resulta difícil obtener conclusiones claras sobre el papel de las variables sociodemográficas, el estudio de la relación entre estas variables y el burnout se plantea de forma exploratoria en el presente estudio.

\section{MÉTODO}

\section{Participantes}

La selección de los participantes se llevó a cabo mediante un muestreo estratificado de docentes en el municipio de Benidorm. Se crea- 
ron estratos según las zonas del municipio (tres zonas escolares), por etapas educativas (Educación Infantil, Primaria, ESO y Bachillerato) y según el tipo de centro (público, privado concertado y privado no concertado). Así la muestra de docentes debía representar a la totalidad de tipos de centros, zonas geográficas del municipio y etapas educativas. La muestra estuvo compuesta por un total de 171 docentes, lo que representaba el 20.12\% de la población total de docentes de Benidorm (850), de los cuales un $72.5 \%$ eran mujeres $(n=124)$ y un $27.5 \%$ eran varones $(n=47)$. El rango de edad del total de la muestra estaba comprendido entre 22 y 64 años, con una edad media de 39.23 (DT = 11.12). La edad media era similar para el grupo de hombres que para el de mujeres $(t=.99$, $p>.05)$. Las restantes características sociodemográficas de la muestra se indican en la Tabla 1. Como puede apreciarse en dicha tabla, en la muestra predominaban los docentes que trabajaban en el nivel de Primaria y en centros públicos, y que tenían un trabajo fijo y eran no practicantes desde el punto de vista religioso. Un $26.3 \%$ de los participantes eran católicos practicantes; el resto estaba compuesto por católicos no practicantes, indiferentes, ateos, agnósticos, y creyentes de otra religión (1.2\%).

\section{Instrumentos de evaluación}

Cuestionario Socio-demográfico y Laboral $(C S L)$. Este cuestionario, aparte de utilizarse para recoger la información sobre las diversas variables socio-demográficas (sexo, edad, tipo de centro, etapa educativa, etc.), incluye las escalas de Satisfacción laboral y Problemática laboral. La escala de Satisfacción laboral está constituida por 15 items que evalúan el grado de satisfacción con una serie de aspectos relacionados con su trabajo, centro y profesión, según una escala Likert de 4 puntos que varía entre 1 ("Muy insatisfecho") y 4 ("Muy satisfecho") (rango 15-60) (véase el Anexo I). La escala de Problemática laboral incluye 9 items que evalúan hasta qué punto diversos aspectos del trabajo (p.ej., mantener la disciplina en el aula) constituyen un problema para el profesor, según una escala Likert de 2 puntos que varía
Tabla 1. Características socio-demográficas de la muestra $(N=171)$

\begin{tabular}{|c|c|c|}
\hline & $\begin{array}{c}\text { Frecuencia } \\
(\%)\end{array}$ & $\chi^{2}$ (g.1.) \\
\hline \multicolumn{3}{|l|}{ Etapa educativa } \\
\hline Infantil & $34(19.9 \%)$ & \\
\hline Primaria & $93(54.4 \%)$ & $34.98 *(2)$ \\
\hline ESO y bachillerato & $44(25.7 \%)$ & \\
\hline \multicolumn{3}{|l|}{ Tipo de centro } \\
\hline Público & $127(74.3 \%)$ & \\
\hline Privado concertado & $12(7.0 \%)$ & $132.56^{*}(2)$ \\
\hline Privado no concertado & $32(18.7 \%)$ & \\
\hline \multicolumn{3}{|l|}{ Sexo } \\
\hline Varón & $47(27.5 \%)$ & $34.67 *(1)$ \\
\hline Mujer & $124(72.5 \%)$ & \\
\hline \multicolumn{3}{|l|}{ Estado civil } \\
\hline Soltero/a & $75(43.9 \%)$ & \\
\hline Casado/a & $86(50.3 \%)$ & \\
\hline Divorciado/a/viudo/a & $10(5.8 \%)$ & $59,19 *(2)$ \\
\hline \multicolumn{3}{|l|}{ Relaciones de pareja } \\
\hline Con pareja & $133(77.8 \%)$ & $52.78 *(1)$ \\
\hline Sin pareja & $38(22.2 \%)$ & \\
\hline \multicolumn{3}{|l|}{ Hijos } \\
\hline Sin hijos & $96(56.1 \%)$ & $2.58(1)$ \\
\hline Con hijos & $75(43.9 \%)$ & \\
\hline \multicolumn{3}{|l|}{ Situación laboral } \\
\hline Eventual & $37(21.6 \%)$ & $55.02 *(1)$ \\
\hline Fijo & $134(78.4 \%)$ & \\
\hline \multicolumn{3}{|l|}{ Religión } \\
\hline No practicante & $126(73.7 \%)$ & $38.37 *(1)$ \\
\hline Católico practicante & $45(26.3 \%)$ & \\
\hline \multicolumn{3}{|l|}{ Años en la profesión } \\
\hline 0-10 años & $86(50.3 \%)$ & $23.19 *(2)$ \\
\hline 11-20 años & $37(21.6 \%)$ & \\
\hline 21-40 años & $48(28.1 \%)$ & \\
\hline
\end{tabular}

entre 1 ("No es problema") y 3 ("Es un problema principal") (rango $=9-27$ ) (véase el Anexo II). El CSL fue elaborado específicamente para el presente estudio y su construcción se basó en estudios previos sobre satisfacción laboral y problemática relacionada con el trabajo docente (Moya-Albiol, Serrano y Salvador, 2010; Skaalvik y Skaalvik, 2009; Veldman, Tartwijk, Brekelmans y Wubbels, 2013). En el presente estudio, los coeficientes de consistencia interna (alfa de Cronbach) para estas dos escalas fueron de .81 (satisfacción laboral) y .79 (problemática laboral). 
Cuestionario de Burnout del Profesorado-Revisado (CBP-R). El CBP-R (Moreno, Garrosa, y González, 2000) es un cuestionario de 66 items que evalúa los procesos de estrés y burnout específicos de la profesión docente, así como también ciertas variables que podrían actuar como antecedentes de estos procesos. El cuestionario evalúa tres áreas generales (estrés y burnout, desorganización, y problemática administrativa) que, a su vez, incluyen diversas escalas (8 escalas en total). El área de Estrés y burnout incluye las escalas de Estrés de rol (13 items; p.ej., "Intentar impedir que mi trabajo sea demasiado rutinario y aburrido me causa mucho estrés"), Agotamiento emocional (8 items; p.ej., "Siento que mi trabajo está afectando negativamente mi salud"), Despersonalización (4 items; p.ej., "Siento que mis alumnos son el enemigo") y Falta de realización (7 items; "Actualmente encuentro que mi vida es muy provechosa"; esta variable se codifica de forma inversa). El área de Desorganización incluye las escalas de Supervisión (12 items; "Mi supervisor da la cara por la gente que trabajamos en el instituto; ítem inverso) y Condiciones organizacionales (9 items; "Los padres están implicados en la educación de sus hijos"; ítem inverso). Finalmente, el área de Problemática administrativa incluye las escalas de Preocupaciones profesionales (9 items; "Conflictos con la administración") y Falta de reconocimiento profesional (4 items; "Salario bajo"). Los 11 primeros items CBP-R se responden según una escala de grado de afectación que varía entre 1 ("No me afecta") y 5 ("Me afecta muchísimo"). Los restantes items según una escala sobre el grado de acuerdo que varía entre 1 ("Totalmente en desacuerdo") y 5 ("Totalmente de acuerdo"). Las propiedades psicométricas del cuestionario son aceptables y han sido publicadas por los autores (Moreno et al., 2000). En lo que concierne al presente estudio, salvo para las escalas de condiciones organizacionales y despersonalización, los coeficientes alfa de Cronbach fueron $\geq .78$ (véase la Tabla 2).

Escalas de Afecto Positivo y Negativo [Positive and Negative Affect Schedule, PANAS] (Watson,Clark y Tellegen, 1988). Se aplicó la versión española adaptada por Sandín et al. (1999). Consta de 20 items, 10 referidos al afec- to positivo y 10 al afecto negativo. Cada ítem se puntúa según una escala Likert de intensidad que varía entre 1 («Nada o casi nada») a 5 («Muchísimo»). Las propiedades psicométricas de esta versión son excelentes (Nolla, Queral, y Miró, 2014; Sandín et al., 1999). En el presente estudio, los coeficientes alfa de Cronbach fueron de .92 para la escala de Afecto positivo y .85 para la escala de Afecto negativo.

\section{Procedimiento}

Los protocolos fueron aplicados, tras consentimiento informado por los profesores que voluntariamente decidieron participar en el estudio, en 18 centros educativos del municipio de Benidorm (Alicante). La selección de los centros se llevó a cabo al azar a partir de las principales zonas escolares de Benidorm, incluyendo las diferentes etapas educativas (infantil, primaria, ESO y bachillerato) y los distintos tipos de centros (público, privado concertado, y privado no concertado). El contacto con los profesores participantes se llevó a cabo a través de los directores de los centros, los cuales informaron a los profesores sobre las características de la investigación y entregaron el protocolo con las instrucciones correspondientes a los que aceptaron colaborar. En todo momento, tanto los directores de los centros como los participantes podían contactar directamente con el primer autor para clarificar cualquier duda que pudiesen tener durante la cumplimentación de los protocolos.

\section{RESULTADOS}

\section{Estadísticos descriptivos y diferencias entre varones y mujeres en las variables del estudio}

En la Tabla 2 se indican los valores correspondientes a las medias y las desviaciones típicas en función del sexo. También se indican los coeficientes alfa de Cronbach para las variables del estudio (los datos sobre las variables sociodemográficas se indican en la Tabla 1). Como se refleja a partir de los resultados de la prueba de $F$ (ANOVA de un factor), únicamente encontramos diferencias significativas entre hombres y muje- 
res en las variables de despersonalización y falta de reconocimiento profesional. En ambos casos, los hombres puntuaron de forma más elevada que las mujeres. No obstante, estas diferencias dejaron de ser significativas estadísticamente tras aplicar la prueba de corrección de Bonferroni.

Tabla 2. Medias (DTs) en las variables laborales en función del sexo

\begin{tabular}{|c|c|c|c|c|c|}
\hline & $\begin{array}{c}\text { Total } \\
(N=171)\end{array}$ & $\begin{array}{l}\text { Hombres } \\
(n=47)\end{array}$ & $\begin{array}{l}\text { Mujeres } \\
(n=124)\end{array}$ & $\begin{array}{c}\text { Hombres vs. } \\
\text { Mujeres }\end{array}$ & \\
\hline & Media $(D T)$ & Media $(D T)$ & Media $(D T)$ & $F(1.169)$ & $\alpha$ \\
\hline \multicolumn{6}{|l|}{ CSL } \\
\hline Satisfacción laboral & $43.14(5.65)$ & $42.28(5.90)$ & $43.47(5.55)$ & 1.4 & .81 \\
\hline Problemática laboral & $16.06(4.27)$ & $16.34(4.51)$ & $15.96(4.18)$ & $<1$ & .79 \\
\hline \multicolumn{6}{|l|}{ CBP-R } \\
\hline Agotamiento emocional & $17.71(6.17)$ & $19.0(6.27)$ & $17.23(6.09)$ & 2.53 & .86 \\
\hline Despersonalización & $6.67(2.39)$ & $7.34(2.51)$ & $6.41(2.30)$ & $5.28 *$ & .66 \\
\hline Falta de realización & $14.49(4.74)$ & $15.00(4.77)$ & $14.30(4.73)$ & $<1$ & .82 \\
\hline Estrés de rol & $34.18(8.34)$ & $35.72(8.09)$ & $33.59(8.38)$ & 1.84 & .82 \\
\hline Supervisión & $25.99(7.23)$ & $26.79(6.81)$ & $25.69(7.39)$ & 1.15 & .78 \\
\hline Condiciones organizacionales & $21.90(4.16)$ & $22.26(3.83)$ & $21.77(4.28)$ & $<1$ & .34 \\
\hline Preocupaciones profesionales & $18.34(8.23)$ & $19.72(8.65)$ & $17.81(8.04)$ & 1.70 & .83 \\
\hline Falta reconocimiento profesional & $10.95(4.06)$ & $12.04(3.90)$ & $10,54(4.05)$ & $4.78^{*}$ & .89 \\
\hline \multicolumn{6}{|l|}{ PANAS } \\
\hline Afecto positivo & $32.71(7.37)$ & $33.45(7.24)$ & $32.42(7.41)$ & $<1$ & .92 \\
\hline Afecto negativo & $18.64(8.23)$ & $18.57(5.61)$ & $18.67(5.36)$ & $<1$ & .85 \\
\hline
\end{tabular}

Nota. CSL $=$ Cuestionario Sociodemográfico y Laboral; CBP-R $=$ Cuestionario de Burnout del Profesorado-Revisado; PANAS $=$ Positive and Negative Affect Schedule. ${ }^{*} p<.05$ ( $p>.05$ tras aplicar la corrección de Bonferroni).

Con el fin de comparar las puntuaciones del CBP-R obtenidas en el presente estudio con las puntuaciones referidas por Moreno et al. (2000), transformamos las puntuaciones directas en puntuaciones en un rango entre 1 y 5 (dividiendo la puntuación directa por el número de items de cada escala del CBP-R). Los resultados se presentan en la Tabla 3, y denotan niveles bajos de burnout y problemas organizacionales. Aunque los valores tienden a ser ligeramente superiores en el estudio de Moreno et al. (2000), en general las puntuaciones son muy similares. En ambos estudios las mayores puntuaciones corresponden a la falta de reconocimiento profesional y al estrés de rol, mientras que las más bajas se dan para la despersonalización y las preocupaciones profesionales.

Tabla 3. Medias y DTs en las variables del CBP-R correspondientes al presente estudio y al estudio de Moreno et al. (2000). Las puntuaciones han sido estandarizadas a un rango de 1-5

\begin{tabular}{lcc}
\hline & Estudio actual & Estudio de Moreno et al. (2000) \\
\hline Variable del CBP-R & Media (DT) & Media $(D T)$ \\
Agotamiento emocional & $2.21(.77)$ & $2.22(.70)$ \\
Despersonalización & $1.67(.59)$ & $1.65(.53)$ \\
Falta de realización personal & $2.07(.67)$ & $2.20(.63)$ \\
Estrés de rol & $2.63(.64)$ & $2.73(.62)$ \\
Supervisión & $2.17(.60)$ & $2.66(.68)$ \\
Condiciones organizacionales & $2.43(.46)$ & $2.61(.56)$ \\
Preocupaciones profesionales & $2.04(.91)$ & $2.15(.74)$ \\
Falta de reconocimiento profesional & $2.74(.99)$ & $3.03(.89)$ \\
\hline
\end{tabular}


Predicción de las puntuaciones en las dimensiones de burnout por las variables sociodemográficas

Con objeto de examinar el posible papel de las variables sociodemográficas como predictores de las puntuaciones en las dimensiones de burnout, aplicamos un diseño de análisis de regresión múltiple por pasos sucesivos para cada una de las tres variables dependientes (agotamiento emocional, despersonalización, y falta de realización personal). El diseño incluía como predictores las variables sociodemográficas siguientes (véase la Tabla 1): edad, sexo, etapa educativa, tipo de centro, relaciones de pareja, hijos, situación laboral, religión y años de profesión. Mediante estos análisis pretendíamos determinar qué variables sociodemográficas eran relevantes para predecir las puntuaciones en burnout.
Los resultados de estos análisis se indican en la Tabla 4. Tal y como puede apreciarse en dicha tabla, la variable religión (ser o no católico practicante) resultó ser un predictor significativo del nivel de burnout en las tres dimensiones de burnout, explicando de forma negativa el $7 \%$ de la varianza en agotamiento emocional y el 5\% de la varianza en falta de realización personal, e incrementando en un 3\% la varianza explicada en las puntuaciones en despersonalización por la variable etapa educativa. Esta última variable predijo de forma positiva el nivel de despersonalización, explicando el $4 \%$ de la varianza (primer paso). Por tanto, de estos análisis se deduce que sólo estas dos variables sociodemográficas resultaron ser predictores significativos de las puntuaciones en burnout, siendo la práctica religiosa (ser católico practicante) un factor protector del burnout y el mayor grado de la etapa educativa un factor de riesgo.

Tabla 4. Predicción de las puntuaciones en las dimensiones de burnout (agotamiento emocional, despersonalización, y falta de realización personal) a partir de las variables sociodemográficas. Análisis de regresión de pasos sucesivos

\begin{tabular}{|c|c|c|c|c|c|c|}
\hline & $R^{2}$ & $\Delta R^{2}$ & $B$ & ET B & Beta & $t$ \\
\hline $\begin{array}{l}\text { Agotamiento emocional } \\
\text { Paso 1: Religión }\end{array}$ & $.07 * * *$ & $.07 * * *$ & -3.77 & 1.03 & -.27 & $-3.65 * * *$ \\
\hline $\begin{array}{l}\text { Despersonalización }^{a} \\
\text { Paso 1: Etapa educativa } \\
\text { Paso 2: Religión }\end{array}$ & $\begin{array}{l}.04^{*} \\
.07 * *\end{array}$ & $\begin{array}{l}.04 * \\
.03 *\end{array}$ & $\begin{array}{l}.66 \\
-.95\end{array}$ & $\begin{array}{l}.26 \\
.40\end{array}$ & $\begin{array}{l}.19 \\
-.18\end{array}$ & $\begin{array}{l}2.49^{*} \\
-2.34^{*}\end{array}$ \\
\hline $\begin{array}{l}\text { Falta de realización personal } \\
\text { Paso 1: Religión }\end{array}$ & $.05^{* *}$ & $.05 * *$ & -.27 & .80 & -.21 & $-2.81 * *$ \\
\hline
\end{tabular}

Nota. Las variables dependientes se indican en cursiva. ${ }^{a}$ Los estadísticos referidos a los coeficientes $B$ y beta corresponden al último paso. $* p<.05, * * p<.01, * * * p<.001$.

\section{Predicción de las puntuaciones en las dimensiones de burnout por las variables organizacionales}

En una segunda fase procedimos de modo similar para seleccionar las variables relevantes correspondientes al contexto organizacional (satisfacción laboral, problemática laboral, estrés de rol, supervisión, etc.). Las correlaciones entre las variables organizacionales y las puntuaciones en las variables de burnout se indican en la Tabla 5. Como cabría esperar, tanto la satisfacción laboral como el afecto positivo correlacionan negativamente y de forma mode- rada-alta, mientras que el resto de variables lo hacen de forma positiva. Entre las variables que correlacionan de forma positiva con las puntuaciones en burnout, destacan por el mayor tamaño del efecto el estrés de rol, el afecto negativo, y las condiciones organizacionales. La correlaciones más bajas del burnout se evidencian con la problemática laboral y las preocupaciones profesionales (véase la Tabla 5).

Para seleccionar las variables organizacionales relevantes aplicamos un diseño de análisis de regresión de pasos sucesivos para cada dimensión de burnout, incluyendo en la ecuación de regresión como variables independientes las 
7 variables organizacionales (evaluadas a través del CSL y el CBP-R; véase la Tabla 5). Los resultados de estos análisis se indican en la Tabla 6.

Tabla 5. Correlaciones de Pearson entre las variables laborales y de personalidad y las dimensiones de burnout

\begin{tabular}{|c|c|c|c|}
\hline & $\begin{array}{c}\text { Agotamiento } \\
\text { emocional }\end{array}$ & Despersonalización & $\begin{array}{c}\text { Falta de realización } \\
\text { personal }\end{array}$ \\
\hline \multicolumn{4}{|l|}{ CSL: } \\
\hline Satisfacción laboral & $-.53 * * *$ & $-.40 * * *$ & $-.53 * * *$ \\
\hline Problemática laboral & $.17^{*}$ & .14 & .14 \\
\hline \multicolumn{4}{|l|}{ CBP-R: } \\
\hline Estrés de rol & $.78 * * *$ & $.50 * * *$ & $.53 * * *$ \\
\hline Supervisión & $.38 * * *$ & $.25^{* *}$ & $.43 * * *$ \\
\hline Condiciones organizacionales & $.47 * * *$ & $.42 * * *$ & $.41 * * *$ \\
\hline Preocupaciones profesionales & $.23 * *$ & $.18^{*}$ & $.15^{*}$ \\
\hline Falta de reconocimiento profesional & $.38 * * *$ & $.23 * *$ & $.20 * *$ \\
\hline \multicolumn{4}{|l|}{ PANAS: } \\
\hline Afecto positivo & $-.43 * * *$ & $-.39 * * *$ & $-.59 * * *$ \\
\hline Afecto negativo & $.54 * * *$ & $.44 * * *$ & $.46^{* * *}$ \\
\hline
\end{tabular}

Nota. CSL $=$ Cuestionario Sociodemográfico y Laboral; CBP-R $=$ Cuestionario de Burnout del Profesorado-Revisado; PANAS $=$ Positive and Negative Affect Schedule. $* p<.05, * * p<.01, * * * p<.001$.

En la Tabla 6 presentamos los resultados de esta serie de análisis de regresión, los cuales indican que dos variables organizacionales parecen ejercer un efecto particularmente relevante sobre el burnout, i.e., las variables de estrés de rol y satisfacción laboral. Ambas variables predijeron de forma significativa las puntuaciones en las tres dimensiones de burnout. El estrés de rol fue seleccionada siempre en primer lugar en los tres análisis de regresión. Adicionalmente, la variable referida a las condiciones organizacionales también predijo significativamente la despersonalización, y la variable de supervisión predijo la falta de realización personal. El resto de variables organizacionales (problemática laboral, preocupaciones profesionales, y falta de reconocimiento profesional) resultaron inefectivas para predecir los niveles de burnout. Cabe resaltar que, especialmente en relación con el agotamiento emocional, el porcentaje de varianza explicada por los modelos de regresión fue elevado (el 63\% de la varianza en agotamiento emocional era explicado conjuntamente por el estrés de rol y la satisfacción laboral).

Tabla 6. Predicción de las puntuaciones en las dimensiones de burnout (agotamiento emocional, despersonalización, y falta de realización personal) a partir de las variables organizacionales. Análisis de regresión de pasos sucesivos

\begin{tabular}{|c|c|c|c|c|c|c|}
\hline & $R^{2}$ & $\Delta R^{2}$ & $B$ & ET B & Beta & $t$ \\
\hline \multicolumn{7}{|l|}{ Agotamiento emocional } \\
\hline Paso 1: Estrés de rol & $.60 * * *$ & $.60 * * *$ & .50 & .04 & .68 & $12.72 * * *$ \\
\hline Paso 2: Satisfacción laboral & $.63 * * *$ & $.03 * * *$ & -3.25 & .87 & -.20 & $-3.72 * * *$ \\
\hline \multicolumn{7}{|l|}{ Despersonalización } \\
\hline Paso 1: Estrés de rol & $.25 * * *$ & $.25 * * *$ & .10 & .02 & .35 & $4.35 * * *$ \\
\hline Paso 2: Condiciones organizacionales & $.28 * * *$ & $.03 * *$ & .10 & .04 & .17 & $2.14^{*}$ \\
\hline Paso 3: Satisfacción laboral & $.30 * * *$ & $.02 *$ & -.91 & .49 & -.15 & $-1.87^{*}$ \\
\hline \multicolumn{7}{|l|}{ Falta de realización personal } \\
\hline Paso 1: Estrés de rol & $.28 * * *$ & $.28 * * *$ & .18 & .04 & .32 & $4.53 * * *$ \\
\hline Paso 2: Satisfacción laboral & $.38 * * *$ & $.10 * * *$ & -3.80 & .90 & -.30 & $-4.19 * * *$ \\
\hline Paso 3: Supervisión & $.40 * * *$ & $.02 *$ & .11 & .04 & .16 & $2.26^{*}$ \\
\hline
\end{tabular}

Nota. Las variables dependientes se indican en cursiva. Los estadísticos referidos a los coeficientes $B$ y beta corresponden al último paso. $* p<.05, * * p<.01, * * * p<.001$. 
Predicción de las puntuaciones en las dimensiones de burnout: Modelos predictivos finales

Finalmente, llevamos a cabo un análisis de regresión múltiple incluyendo en el modelo todas las variables seleccionadas a partir de los análisis anteriores (indicados en las Tablas
4 y 6) junto con las variables de personalidad (afecto positivo y negativo). De este modo, no sólo podremos conocer la posible influencia de la afectividad, sino también la relevancia de las variables organizacionales controlando el efecto de la afectividad. Los resultados de estos análisis están indicados en la Tabla 7.

Tabla 7. Modelos predictivos finales de las puntuaciones en las dimensiones de burnout. Análisis de regresión múltiple con inclusión simultánea de las variables independientes

\begin{tabular}{|c|c|c|c|c|c|c|}
\hline & $R^{2}$ & $B$ & $E T B$ & Beta & $t$ & $r$ parcial \\
\hline \multicolumn{7}{|l|}{ Agotamiento emocional } \\
\hline Afecto positivo & $.66 * * *$ & -.07 & .04 & -.09 & -1.65 & -.13 \\
\hline Afecto negativo & & .17 & .06 & .15 & $2.67 * *$ & .21 \\
\hline Religión & & -.76 & .67 & -.06 & -1.14 & -.09 \\
\hline Estrés de rol & & .42 & .04 & .57 & $9.47 * * *$ & .59 \\
\hline Satisfacción laboral & & -2.71 & .87 & -.17 & $-3.10 * *$ & -.24 \\
\hline \multicolumn{7}{|l|}{ Despersonalización } \\
\hline Afecto positivo & $.38 * * *$ & -.05 & .02 & -.17 & $-2.43^{*}$ & -.19 \\
\hline Afecto negativo & & .10 & .03 & .20 & $2.64 * *$ & .21 \\
\hline Etapa educativa & & .50 & .22 & .14 & $2.26^{*}$ & .18 \\
\hline Religión & & -.10 & .35 & -.02 & -.27 & -.02 \\
\hline Estrés de rol & & .05 & .02 & .19 & $2.18^{*}$ & .17 \\
\hline Satisfacción laboral & & -.64 & .48 & -.10 & -1.32 & -.11 \\
\hline Condiciones organizacionales & & .08 & .04 & .15 & $1.98^{*}$ & .15 \\
\hline \multicolumn{7}{|l|}{ Falta de realización personal } \\
\hline Afecto positivo & $.55 * * *$ & -.24 & .03 & -.37 & $-6.16^{* * *}$ & -.43 \\
\hline Afecto negativo & & .16 & .05 & .18 & $2.82 * *$ & .22 \\
\hline Religión & & -.31 & .60 & -.03 & -.51 & -.04 \\
\hline Estrés de rol & & .06 & .04 & .11 & 1.47 & .12 \\
\hline Satisfacción laboral & & -3.07 & .81 & -.25 & $-3.78 * * *$ & -.28 \\
\hline Supervisión & & .09 & .04 & .13 & $2.09 *$ & .16 \\
\hline
\end{tabular}

Nota. Las variables dependientes se indican en cursiva. ${ }^{*} p<.05,{ }^{* *} p<.01, * * * p<.001$.

Como puede observarse siguiendo los datos que presentamos en la Tabla 7, no todas las variables independientes introducidas en los análisis de regresión mantuvieron su poder predictivo sobre las puntuaciones en la correspondiente dimensión de burnout. Las dos variables de personalidad (afecto positivo y afecto negativo) predijeron significativamente las puntuaciones en despersonalización y falta de realización personal, pero sólo el afecto negativo predijo también el agotamiento emocional. El estrés de rol resultó efectivo para predecir el agotamiento emocional y la despersonalización, pero no predijo significativamente la falta de realización personal. La satisfacción laboral predijo el agotamiento emocional y la falta de realización personal, pero inesperadamente no predijo la despersonalización. Las condiciones organizativas (p.ej., falta de implicación de los padres en la educación de los hijos, no tener claras las funciones, recursos inadecuados) favorecen la despersonalización, pero no parecen influir en otras dimensiones del burnout. La supervisión (p.ej., falta de apoyo por parte de la dirección del centro, falta de participación en decisiones del centro) predice únicamente la falta de realización personal.

En lo que concierne a las variables sociodemográficas, aunque ser católico practicante predecía negativamente las puntuaciones en cada una de las tres dimensiones de burnout, al controlar la influencia de las variables de persona- 
lidad y organizacionales perdió su capacidad predictiva, no resultando significativa para ninguna de las dimensiones de burnout. En contraste, la variable relacionada con la etapa educativa mantuvo su poder predictivo tras controlar el efecto de las variables de personalidad y laborales. La etapa educativa predijo de forma positiva el nivel de despersonalización, lo cual indica que cuanto mayor sea el nivel educativo mayor será la puntuación en despersonalización. Así mismo, tras aplicar un ANOVA de un factor encontramos efectos principales de la etapa educativa $\left(F_{2,168}=\right.$ $3.73, p<.05)$, siendo las puntuaciones medias para cada dimensión del factor de $5.74(D T=$ 2.20; Infantil), 6.77 ( $D T=2.31$; Primaria) y 7.16 $(D T=2.51$; ESO y Bachillerato), respectivamente. Mediante la prueba post-hoc de Bonferroni encontramos diferencias estadísticamente significativas $(p<.05)$ en despersonalización entre las etapas de Primaria y ESO/Bachillerato.

\section{DISCUSIÓN}

En la presente investigación hemos estudiado el posible papel de los factores laborales, personales y sociodemográficos como predictores de los niveles de burnout en una muestra de docentes. En relación con los factores específicos del contexto sociolaboral habíamos hipotetizado un efecto protector de los niveles de burnout asociado a la satisfacción laboral, y un efecto negativo (i.e., incremento de los niveles de burnout) relacionado con posibles factores de vulnerabilidad como el estrés de rol, la problemática laboral, la falta de apoyo de supervisión, las condiciones organizacionales inadecuadas (p.ej., participación inadecuada de los padres de los estudiantes), las preocupaciones profesionales, y la falta de reconocimiento profesional. Así mismo, esperábamos una asociación predictiva positiva entre el afecto negativo y los niveles de burnout, y una asociación negativa entre el afecto positivo y el nivel de burnout. Finalmente, y dada la diversidad de resultados relacionados con la implicación de los factores sociodemográficos en relación con el burnout en este tipo de profesionales, no establecimos hipótesis específicas sobre tales factores y los niveles de burnout.
Los resultados del presente estudio reflejan, en primer lugar, que los niveles medios de burnout correspondientes a la muestra general de docentes de la ciudad de Benidorm eran bajos en las tres dimensiones de burnout. En un rango de valores entre 1 y 5 , las puntuaciones medias obtenidas fueron de 2.21 (agotamiento emocional), 1.67 (despersonalización) y 2.07 (falta de realización personal). Las desviaciones típicas oscilaron entre .59 (despersonalización) y .77 (agotamiento emocional). En términos generales, estos resultados son muy similares a los referidos por Moreno et al. (2000) a partir de una muestra de docentes de la ciudad de Madrid.

Respecto al papel de los factores sociolaborales (i.e., factores que caracterizan los aspectos laborales u organizacionales de la actividad docente), aunque examinamos la implicación de un conjunto amplio de factores que potencialmente podrían estar implicados, únicamente encontramos que algunos de ellos parecían predecir de forma significativa las puntuaciones en algunas de las dimensiones de burnout. En principio encontramos que dos factores del entorno laboral, i.e., el estrés de rol (o carga laboral) y la satisfacción laboral, parecían relacionarse de forma consistente con todas y cada una de las tres dimensiones del burnout, pudiendo actuar el primero como factor de vulnerabilidad y el segundo como factor protector (las puntuaciones elevadas en estrés de rol predecían un incremento en las puntuaciones de burnout, mientras que las puntuaciones elevadas en satisfacción laboral predecían un descenso de los niveles de burnout). Aunque desde distintos enfoques, el papel especial que parecen jugar estas dos variables en relación con la ocurrencia de burnout ha sido señalado por diversos autores (Durán et al., 2005; Gil-Monte y Peiró, 1997; Pyhältö, Pietarinen y Salmela-Aro, 2011; Sandín, 2008; Skaalvik y Skaalvik, 2010; Vélez y Mayorga, 2010). En el presente estudio, no obstante, se constata que la relación del estrés laboral y la satisfacción laboral con el burnout no parece ser igual con las tres dimensiones de burnout. Así, tras controlar el efecto de las variables de personalidad y sociodemográficas, el efecto del estrés de rol dejaba de predecir significativamente la falta de realización personal, mientras que la satisfacción laboral resultaba inefectiva para predecir el nivel de despersonalización. 
Si nos atenemos a los resultados de los análisis de regresión, observamos la estrecha relación que parece existir entre el estrés laboral (estrés de rol) y el agotamiento emocional. A partir de nuestro estudio se concluye que el agotamiento emocional depende en gran medida del nivel de estrés relacionado con la actividad docente (p.ej., por cambios en las normas, problemas de los estudiantes, exigencias de la docencia, burocracia, etc.), aunque también se relaciona con el nivel de satisfacción laboral (p.ej., satisfacción con los horarios de trabajo, los medios didácticos, las expectativas de promoción, el desarrollo de la profesión, etc.). Aunque el presente estudio es de tipo transversal y no posibilita establecer relaciones causales, los datos tienden a indicar que el estrés laboral constituye un factor fundamental de vulnerabilidad del cansancio emocional (sin duda la dimensión más relevante del burnout). Así mismo, y con la salvedad indicada, podríamos concluir diciendo que la satisfacción laboral podría conceptualizarse como un factor significativamente protector del cansancio emocional.

Como indicamos arriba, las dos dimensiones restantes del burnout (despersonalización y falta de realización personal) se asocian diferencialmente al estrés laboral y la satisfacción laboral. La dimensión de despersonalización se asoció de forma específica con el estrés relacionado con la actividad docente; no obstante, la escala de despersonalización posee escasa consistencia interna (alfa $=.66$ ), por lo cual estos resultados deben tomarse con cierta cautela. La dimensión de falta de realización personal fue predicha de forma negativa y significativa por la satisfacción laboral (la satisfacción laboral fomentaría la realización personal), y de forma positiva por la carencia de supervisión de apoyo (p.ej., falta de apoyos del director del centro, participación en las decisiones, etc.). Esta relación con el deterioro de la realización personal es esperable conceptualmente partiendo del significado de ambos constructos (satisfacción laboral y falta de apoyos de supervisión). Estos resultados contrastan con la evidencia presentada recientemente por Skaalvik y Skaalvik (2010) sobre la relación significativa de la satisfacción laboral y el apoyo de supervisión con la despersonalización.
Aunque los estudios sobre la implicación de las variables de personalidad en el burnout en profesionales de la docencia son escasos, la evidencia de la literatura tiende a indicar que la afectividad negativa (o neuroticismo) se asocia de forma positiva con el burnout, mientras que la afectividad positiva (y la extraversión) se relacionan de forma negativa (Reichl, Wach, Spinath, Brünken y Karbach, 2014; Sandín, 2008). Dicho en otros términos, mientras que el afecto negativo constituye un factor de vulnerabilidad para experimentar burnout, el afecto positivo parece actuar como factor protector. No obstante, nuestros resultados indican que el afecto negativo se relaciona con el burnout de forma más consistente que el afecto positivo. En los modelos finales de regresión, el afecto negativo predijo significativamente las tres dimensiones de burnout, mientras que el afecto positivo resultó inefectivo para predecir el agotamiento emocional (principal dimensión del burnout). Estos resultados confirman la especial relación del estrés y la afectividad negativa con el agotamiento emocional, o en términos más amplios con el denominado agotamiento vital (Sandín, 2002, 2008), el cual ha sido asociado de forma consistente con la cardiopatía coronaria (Montero, Rueda y Bermúdez, 2012; Sandín, 2002).

Los resultados obtenidos en el presente estudio en relación con la implicación de las variables sociodemográficas son poco consistentes y permiten extraer escasas conclusiones al respecto. Aunque la religión (ser católico practicante) resultó ser efectiva para predecir las puntuaciones en cada una de las tres dimensiones de burnout (actuando como factor protector) tras controlar la influencia de las restantes variables sociodemográficas, dejó de ser significativa cuando también se controlaron las variables organizacionales (estrés de rol, satisfacción laboral, condiciones organizacionales, y supervisión) y de personalidad (afecto positivo y afecto negativo). La práctica religiosa ha sido señalada como una forma adaptativa de afrontamiento del estrés (Mytko y Knight, 1999; Sandín y Chorot, 2003), aunque, de acuerdo con los resultados del actual estudio, su papel como factor protector del burnout no queda claro. La única variable sociodemográfica que resultó efectiva para predecir el burnout (dimensión de 
despersonalización), tras haber controlado el efecto de todas las restantes variables incluidas en el estudio, fue la etapa educativa. A este respecto, nuestros datos indican que el mayor nivel de la etapa educativa (no universitaria) en la que trabaje el docente constituye un factor de riesgo de despersonalización (i.e., predicción positiva entre el nivel de la etapa educativa y las puntuaciones en despersonalización). Esta conclusión se obtuvo igualmente al comparar las puntuaciones obtenidas en despersonalización entre los tres grupos (etapas educativas), al constatar puntuaciones significativamente más altas asociadas a la etapa superior (ESO/Bachillerato). Tales resultados confirman la hipótesis sugerida por Beer y Beer (1992) de que el burnout se asocia de forma más específica con las etapas educativas superiores.

Otras variables sociodemográficas como la edad, el sexo, los años en la profesión, el estado civil o las relaciones de pareja, etc., aunque a veces han sido sugeridas como variables relevantes para el burnout en los docentes, no parecen desempeñar un papel significativo en este sentido. Otros autores tampoco han constatado relaciones significativas entre estas variables y las puntuaciones en burnout (Vélez y Mayorga, 2013). Es posible que la discrepancia entre unos estudios y otros se deba a que en algunos de estos no se controlase suficientemente el efecto de terceras variables, dando lugar erróneamente a relaciones significativas.

En suma, los modelos predictivos finales indican que sólo algunas de las variables estudiadas predicen significativamente las puntuaciones de los docentes en burnout. Cabe indicar, como punto de partida, que el afecto negativo parece actuar como predictor general del burnout, asociándose a un incremento del nivel de burnout con independencia de la dimensión de que se trate, pudiendo actuar, por tanto, como un factor de vulnerabilidad general hacia el burnout. El estrés laboral (estrés de rol), así como también las condiciones organizativas inadecuadas y la falta de apoyos de supervisión, parecen constituir factores de riesgo significativos relacionados con la propia organización y actividad docentes. Finalmente, aunque obtuvimos una tendencia hacia puntuaciones superiores en despersonalización en el grupo de varo- nes, las diferencias no fueron significativas. La única variable sociodemográfica que reflejó ser un factor de vulnerabilidad fue el nivel educativo (etapa educativa) en el que se desarrollaba la docencia, constituyendo el nivel más alto (ESO y Bachillerato) un factor de vulnerabilidad hacia la despersonalización.

El presente estudio presenta las limitaciones que corresponden a toda investigación de tipo transversal. Por ello, aunque en el artículo nos referimos a factores de vulnerabilidad y a factores protectores, estos conceptos debemos entenderlos únicamente de forma genérica y sin pretender que necesariamente signifiquen relaciones de causalidad, para lo cual sería necesario aplicar diseños de tipo longitudinal donde pudiesen cuantificarse los efectos causales determinados en el tiempo (cambios en las puntuaciones en burnout) por la influencia de las variables relevantes (variables organizacionales, de personalidad y sociodemográficas). No obstante, merece la pena señalar que en el presente estudio se han aislado variables relevantes que podrían ser evaluadas en futuros estudios longitudinales.

\section{REFERENCIAS}

Beer, J., \& Beer, J. (1992), Burnout and stress, depression and self-esteem of teachers. Psychological Reports, 71, 1331-1336.

Boydak, M. (2009). A study on primary school teacher burnout levels: The Northern Cyprus case. Education, 129, 692-703.

Briones, E., Tabernero C., \& Arenas A. (2010). Job satisfaction of secondary school teachers: Effects of demographic and psycho-social factors. Revista de Psicología del Trabajo y las Organizaciones, 26, 115122.

Burke, R. J., \& Greenglass, E. R. (1994). Towards an understanding of work satisfactions and amotional well-being of school based educators. Stress Medicine, 10, 177-184.

Campagne, D. M. (2012). When therapist run out of steam: Professional boredom or burnout? Revista de Psicopatología y Psicología Clínica, 17, 75-85.

Durán, M. A., Extremera, N., Montalbán, F. M., y Rey, L. (2005). Engagement y burnout en el ámbito docente: Análisis de sus relaciones con la satisfacción laboral y vital en una muestra de profesores. Revista de psicología del trabajo y las organizaciones, 21, 145158. 
Freudenberger, H. J. (1974). Staff Burn-out. Journal of Social Issues, 30, 159-165.

Gascón, S., Olmedo Montes, M., \& Ciccotelli, H. (2003). La prevención del burnout en las organizaciones: el chequeo preventivo de Leiter y Maslach. Revista de Psicopatología y Psicología Clínica, 8, 55-66.

Gil-Monte, P. y Peiró, J. M. (1997). Desgaste psíquico en el trabajo: el síndrome de quemarse. Madrid: Síntesis.

Gómez-Alcaina, B., Montero-Marín, J., Demarzo, M. M., Pereira, J. P., \& García-Campayo, J. (2013). Utilidad de los marcadores biológicos en la detección precoz y prevención del síndrome de burnout. Revista de Psicopatología y Psicología Clínica, 18, 245-253.

González-Morales, M. G., Rodríguez, I., \& Peiró, J. M. (2010). A longitudinal study of coping and gender in a female-dominated occupation: Predicting teachers' burnout. Journal of Occupational Health Psychology, 15, 29-44.

Lau, P. S. Y., Yuen, M. T., \& Chan, R.M.C. (2005). Do demographic characteristics make a difference to burnout among Hong Kong secondary school teachers? Social Indicators Research, 71, 491-516.

León-Rubio, J. M., León-Pérez, J. M., y Cantero, F. J. (2013). Prevalencia y factores predictivos del burnout en docentes de la enseñanza pública: El papel del género. Ansiedad y Estrés, 19, 11-25.

Manassero, A., García, E., Torrens, G., Ramis, C., Vázquez, A., y Ferrer, V. (2005). Burnout en la enseñanza: Aspectos atribucionales. Revista de Psicología del Trabajo y de las Organizaciones, 21, 89-105.

Maslach, C. (1982). Burnout: the cost of caring. Englewood Cliffs, NJ: Prentice-Hall.

Maslach, C., \& Jackson, S.E. (1981). The measurement of experienced burnout. Journal of Ocupational Behavior, 12, 99-113.

Montero, P., Rueda, B., y Bermúdez, J. (2012). Relación de la personalidad tipo D y el agotamiento vital con las emociones negativas y el ajuste psicológico a la enfermedad cardiaca. Revista de Psicopatología y Psicología Clínica, 17, 93-106.

Moreno, B., Aranda, C., Aldrete, M.G., Flores, E., y Pozo, E. (2006). Factores psicosociales y burnout en docentes del centro universitario de ciencias de la salud. Investigaciones en Salud, 7, 173-177.

Moreno, B., Garrosa, E., y González, J. L. (2000). Evaluación del estrés y el burnout del profesorado: El CBP-R. Revista de Psicología del Trabajo y las Organizaciones, 16, 331-349.

Moriarty, V., Edmonds, S., Martín, C., \& Blatchford, P. (2001). Teaching young children: Perceived satisfaction and stress. Educational Research, 43, 33-46.

Moya-Albiol, L., Serrano M. A., \& Salvador, A. (2010). Burnout as an important factor in the psychophysiological responses to a work day in teachers. Stress and Health. 26, 382-393.
Mytko, J. J., \& Knight, S. J., (1999). Body, mind and spirit: towards the integration of religiosity and spirituality in cancer quality life research. Psychooncology, 8, 439450.

Nolla, M. C., Queral, R., y Miró, J. (2014). Escalas PANAS de afecto positivo y negativo: Nuevos datos de su uso en personas mayores. Revista de Psicopatología y Psicología Clínica, 19, 15-21.

Oliver, C. (1993). Análisis de la problemática de estrés en el profesorado de enseñanzas medias: El burnout como sindrome especifico. Tesis doctoral. Universidad Autónoma de Madrid.

Padilla, A., Gómez, C., Rodríguez, V., Dávila, M., Avella, C., Caballero, A., Vives, N., Mora, L., Márquez, G., Prieto, Y., Sandoval, N., Cotes, Z., y Hernández, S. (2009). Prevalencia y características del síndrome de agotamiento profesional (SAP) en docentes de tres colegios públicos de Bogotá (Colombia). Revista Colombiana de Psiquiatría, 38, 50-85.

Pines, A., \& Aronson, E. (1988). Career burnout: Causes and cures. New York: Free Press.

Purvanova, R. K., \& Muros, J. P. (2010). Gender differences in burnout: A meta-analysis. Journal of Vocational Behavior, 77, 168-185.

Pyhältö, K., Pietarinen, J., \& Salmela-Aro, K. (2011). Teachereworking-environment fit as a framework for burnout experienced by Finnish teachers. Teaching and Teacher Education 27, 1101-1110.

Reichl, C., Wach, F. S., Spinath, F. M., Brünken, R., \& Karbach, J. (2014). Burnout risk among first-year teacher students: The roles of personality and motivation. Journal of Vocational Behavior, 85, 85-92.

Ramos, F. (1999). El síndrome de burnout. Madrid: Klinik.

Sandín, B. (2002). Papel de las emociones negativas en el trastorno cardiovascular: Un análisis crítico. Revista de Psicopatología y Psicología Clínica, 7, 1-18.

Sandín, B. (2008). Estrés psicosocial: Conceptos y consecuencias clínicas ( $2^{\mathrm{a}}$ ed.). Madrid: Klinik.

Sandín, B., y Chorot, P. (2003). Cuestionario de Afrontamiento del Estrés (CAE): Desarrollo y validación preliminar. Revista de Psicopatología y Psicología Clínica, 8, 39-52.

Sandín, B., Chorot, P., Lostao, L., Joiner, T.E., Santed, M.A., y Valiente, R.M. (1999). Escalas PANAS de afecto positivo y negativo: Validación factorial y convergencia transcultural. Psicothema, 11, 37-51.

Schaufeli, W.B., Leiter, M.P., y Maslach, C. (2009). Burnout: 35 years of research and practice. Career Development International, 14, 204-220.

Schwab, R. L., \& Iwanicki, E. F. (1982). Who are our burned out teachers? Educational Resarch Quarterly, 7, 5-16.

Skaalvik, E. M., \& Skaalvik, S. (2010). Teacher selfefficacy and teacher burnout: A study of relations. Teaching and Teacher Education, 26, 1059-1069. 
Skaalvik, E. M., \& Skaalvik, S. (2009). Does school context matter? Relations with teacher burnout and job satisfaction. Teaching and Teacher Education, 25, 518-524.

Veldman, I., Tartwijk, J., Brekelmans, M, \& Wubbes, T. (2013). Job satisfaction and teacher-studentrelationships across the teaching career: Four cases studies. Teaching and Teacher Education, 32, 55-66.

Vélez, P., y Mayorga, L. D. (2013). Relación de los factores de riesgo psicosociales y el síndrome de burnout en docentes de planta de la Universidad Francisco de Paula Santander en el periodo del 2011-2012. Ciencia y Cuidado, 10, 85-95.

Watson, D., Clark, L. A., \& Tellegen, A. (1988). Development and validation of brief measures of positive and negative affect: the PANAS scales. Journal of Personality and social Psychology, 54, 1063-1070.

\section{APÉNDICE I. ESCALA DE SATISFACCIÓN LABORAL EN LOS DOCENTES}

Instrucciones: Indique el grado de satisfacción que tiene con los aspectos que se indican a continuación relacionados con su centro, su profesión y su actividad laboral. Por favor, rodee con un círculo el número que corresponda.

1

Muy insatisfecho
3

Bastante satisfecho
4

Muy satisfecho

\begin{tabular}{|c|c|c|c|c|}
\hline 1. El centro de trabajo en general & 1 & 2 & 3 & 4 \\
\hline 2. El proyecto educativo & 1 & 2 & 3 & 4 \\
\hline 3. El tipo de alumnos & 1 & 2 & 3 & 4 \\
\hline 4. Los medios didácticos & 1 & 2 & 3 & 4 \\
\hline 5. La colaboración de padres & 1 & 2 & 3 & 4 \\
\hline 6. El prestigio de la profesión & 1 & 2 & 3 & 4 \\
\hline 7. La seguridad y estabilidad en el trabajo & 1 & 2 & 3 & 4 \\
\hline 8. Las posibilidades de usar mi propia iniciativa & 1 & 2 & 3 & 4 \\
\hline 9. Las expectativas de promoción & 1 & 2 & 3 & 4 \\
\hline 10. El desarrollo profesional & 1 & 2 & 3 & 4 \\
\hline 11. La adecuación de la remuneración económica & 1 & 2 & 3 & 4 \\
\hline 12. Los horarios & 1 & 2 & 3 & 4 \\
\hline 13. Las vacaciones & 1 & 2 & 3 & 4 \\
\hline
\end{tabular}

\section{APÉNDICE II. ESCALA DE PROBLEMÁTICA LABORAL EN LOS DOCENTES}

Instrucciones: A pesar de la buena voluntad que suele caracterizar el trabajo docente, a veces resulta difícil evitar problemas y dificultades que surgen en el mismo. indique hasta qué punto le serían aplicables a usted los problemas que se indican a continuación. Por favor, rodee con un círculo el número que corresponda.

1

No es problema
2

Es un problema secundario
3

Es un problema principal

\footnotetext{
1. Coordinación con otros profesores (departamentos, etc.)

2. Disponer de los conocimientos necesarios (formación, actualización)

3. Disponer de los medios didácticos necesarios

4. Ganarme la confianza de los alumnos

5. Mantener la disciplina en el aula

6. Falta de objetivos educativos claros

7. Falta de estímulos económicos

8. Libros de textos inadecuados

9. Falta de colaboración de las familias
}

\begin{tabular}{lll}
1 & 2 & 3 \\
1 & 2 & 3 \\
1 & 2 & 3 \\
1 & 2 & 3 \\
1 & 2 & 3 \\
1 & 2 & 3 \\
1 & 2 & 3 \\
1 & 2 & 3 \\
1 & 2 & 3 \\
\hline
\end{tabular}

